\title{
À propos de la langue et du langage : sensibilisation à la bonne utilisation des dictionnaires
}

Rosalind Greenstein

\section{(2) OpenEdition}

\section{Journals}

Édition électronique

URL : http://journals.openedition.org/asp/2806

DOI : $10.4000 /$ asp.2806

ISSN : 2108-6354

Éditeur

Groupe d'étude et de recherche en anglais de spécialité

\section{Édition imprimée}

Date de publication : 1 décembre 1998

Pagination : 165-185

ISSN : 1246-8185

\section{Référence électronique}

Rosalind Greenstein, « À propos de la langue et du langage : sensibilisation à la bonne utilisation des dictionnaires », ASp [En ligne], 19-22 | 1998, mis en ligne le 13 mars 2012, consulté le 20 avril 2019. URL : http://journals.openedition.org/asp/2806 ; DOI : 10.4000/asp.2806

Ce document a été généré automatiquement le 20 avril 2019.

Tous droits réservés 


\title{
À propos de la langue et du langage: sensibilisation à la bonne utilisation des dictionnaires
}

\author{
Rosalind Greenstein
}

\begin{abstract}
Dictionaries are like watches, the worst is better than none and the best cannot be expected to go quite true. (Samuel Johnson, Lettre à Francesco
\end{abstract}

Sastres, 1784)

\section{Introduction}

1 Mon expérience de l'enseignement de l'anglais de spécialité, en l'occurrence la terminologie juridique anglaise, me conduit depuis quelques années à une certaine réflexion, basée sur un certain nombre de constats. Les étudiants, quelles que soient leurs compétences et leurs connaissances en français, en anglais général et en droit, n'ont jamais, ou très rarement, réfléchi au fonctionnement de la langue/du langage et ne se rendent pas compte de son importance dans leur futur métier. En effet, on peut gagner ou perdre un procès, un client, une réputation si on choisit mal ses mots, si on n'est pas rigoureux dans sa façon de s'exprimer, et ceci dans sa propre langue. A fortiori dans une langue étrangère. Et plus encore, dirai-je, lorsqu'on doit manier une ou deux langues vernaculaires, l'anglais et le français, par exemple, et deux, voire quatre jargons, à savoir l'anglais juridique, le français juridique, et l'anglais et le français communautaires !

2 Nombreux sont ceux qui ne se demandent pas à quel public ils s'adressent, qui se contentent de l'à-peu-près, qui ne savent pas faire la différence entre l'argot, un langage châtié, un jargon hermétique aux non-initiés... Nombreux sont ceux qui pensent qu'il suffit d'apprendre des listes de vocabulaire spécialisé par coeur, sans se soucier un seul instant du «ciment » qu'il faut mettre entre les mots pour qu'ils aient un sens. Et plus d'un a du mal à accepter qu'il n'existe pas toujours d'équivalents car, plus que dans 
d'autres domaines de langue de spécialité, les termes de l'anglais juridique correspondent à des concepts qui dépendent du système. Les listes de vocabulaire comportent donc des trous et, en outre, le ciment dont je viens de parler ne se calque pas parfaitement d'une langue à l'autre. Alors, que font les étudiants non avertis? Ils ont recours aux dictionnaires, souvent généralisés, avec comme résultat des non-sens, barbarismes et autres mauvais choix, leur réponse lorsque je leur demande où ils ont trouvé le terme étant « je l'ai trouvé dans le dictionnaire ».

Mon but est donc de sensibiliser mes étudiants aux questions de langue et à la bonne utilisation des dictionnaires, même si cela reste à un niveau très superficiel. Il faut savoir que je m'adresse surtout aux étudiants en droit (droit des activités économiques ou droit communautaire $-2^{\mathrm{e}}$ et $3^{\mathrm{e}}$ cycles), dont l'attente et les préoccupations sont bien loin de ce que je leur propose comme introduction à la terminologie juridique anglaise. Si possible je consacre, une fois les formalités de début d'année terminée, deux séances de travaux dirigés (environ trois heures) à la réflexion sur la langue et les dictionnaires.

Cette réflexion est basée sur mes propres recherches et se présente à plusieurs niveaux ; c'est par le biais de questions que je pose aux étudiants et par le travail de recherche et d'analyse que je leur demande de faire que j'aborde la matière. Même s'ils sont souvent perplexes à la sortie de la première séance et se demandent ce que cela a à voir avec le droit, ils voient par la suite l'intérêt (dans les deux sens du terme, du moins je l'espère) de cette étude. Surtout que nous passons assez rapidement à la question des sources du droit et aux problèmes d'interprétation que cela comporte. C'est à ce moment-là que la dénotation et la connotation, aspects souvent ignorés des mots, prennent leur place dans les règles d'interprétation littérale, par la logique (apagogique) et téléologique (literal, golden and mischief rules) et que la jurisprudence peut être abordée, qu'elle soit assez ancienne (Heydon's case 1584) ou récente (Pepper $v$ Hart 1993 ; DPP $v$ Bull 1994).

5 Avant de présenter le contenu de cet enseignement, une partie commentée de mes propres recherches ainsi que quelques extraits d'un arrêt, je voudrais préciser que tous les étudiants ne sont pas francophones (exceptionnellement il y a des anglophones) et que, même si les exemples viennent surtout mais pas exclusivement de l'anglais et du français, la discussion se passe entièrement en anglais.

\section{Questions que je pose et réponses des étudiants}

\subsection{Qu'est-ce que le langage?}

6 La première question que je pose paraît souvent saugrenue aux étudiants, mais suscite habituellement un échange d'idées intéressant. Parmi les réponses fréquentes, on trouve : un moyen de communication ; un code, constitué de mots et de règles sur leur utilisation; un moyen d'exprimer les sentiments ou les sensations; un outil typiquement humain (mais que faire de la danse des abeilles ?) ; la communication avec des mots (mais quid du langage des sourds-muets et du langage gestuel/body language ?); un moyen de nommer les choses; un outil de conceptualisation; un moyen de percevoir, de découper, de concevoir et d'ordonner le monde extérieur (Mounin, 1963 : 78).

7 Si les premières réponses leur posent relativement peu de problèmes, la dernière, qui dit que le langage est un moyen de percevoir et d'ordonner le monde (a way of seeing and ordering the world) surprend souvent les étudiants. Ils ont rarement réfléchi au fait qu'un 
mot, de par son existence, reflète et fait exister une perception, une conception du monde, oblige à découper tout ce qui nous entoure, tout ce que nous ressentons, de telle façon et pas de telle autre. Le phénomène n'est évidemment pas purement lexical mais également syntaxique; les langues diffèrent les unes des autres par les informations qu'elles permettent d'exprimer ou de taire, voire qu'elles obligent à exprimer ou taire. Pour mieux illustrer cette idée je propose aux étudiants des exemples que nous analysons ensemble.

\section{Exemple 1}

8 Le mot they. Quelles informations sont contenues dans le mot they en anglais, hors contexte? Comment exprime-t-on la même chose en français?

they (ang.) : pluriel, autre (le locuteur n'en fait pas partie), inanimé (neutre) et/ou animé (uniquement féminin/uniquement masculin/féminin et masculin)

ils (fr.) : pluriel, autre (le locuteur n'en fait pas partie), inanimé (uniquement masculin/féminin et masculin) et/ou animé (uniquement masculin/féminin et masculin)

elles (fr.) : pluriel, autre (le locuteur n'en fait pas partie), inanimé ou animé (uniquement féminin)

9 Le mot anglais they peut être considéré de différentes façons par rapport au français : il est plus général, plus vague, plus ambigu et, si nous nous limitons aux cas animés, recouvre les trois combinaisons possibles, à savoir un groupe entièrement composé d'êtres de sexe féminin, un groupe entièrement composé d'êtres de sexe masculin ou un groupe composé des deux. En revanche, le français est moins général, plus précis, lève une des ambiguïtés car le groupe composé entièrement d'êtres de sexe féminin est clairement identifié par un autre terme. Ce qui ressort de ce petit exemple, c'est qu'il est impossible de traduire le mot they en français en étant sûr de ne pas se tromper, sans un complément d'information.

\section{Exemple 2}

Comment peut-on traduire l'expression l'ouvrier travaille du français en anglais, sans contexte? Quelles sont les informations qui sont contenues dans une des langues mais tues dans l'autre?

\section{français : l'ouvrier travaille}

anglais : a) the worker is working, b) the worker works, c) the worker has been working

11 Dans les trois cas, l'anglais est très clair sur l'aspect temporel et le locuteur doit faire un choix. Nous savons que dans (a) l'action se déroule au moment où l'on parle, dans (b) il s'agit d'une habitude, d'une action répétée et que dans (c) l'action a commencé dans le passé et continue au moment de l'énoncé. Le français, en revanche, ne contient pas ces précisions temporelles et il faudrait d'autres informations pour savoir exactement ce que la périphrase veut dire.

12 Je passe ensuite à un deuxième aspect, le sexe du sujet. Ici l'anglais ne donne aucun élément permettant de savoir s'il s'agit d'un homme ou d'une femme (bien que, soyons clairs, le plus souvent c'est le pronom masculin he qui suivra, sauf si le contexte permet d'affirmer qu'il s'agit d'une femme - mais nous sommes ici dans le domaine culturel, la langue pure n'étant d'aucune aide pour décider). 
Quant au français, les choses sont un peu plus compliquées. Dans les cas (a) et (c) nous parlons d'une personne précise qui travaille au moment où nous parlons et nous savons donc qu'il s'agit d'un homme (sinon nous aurions dit l'ouvrière). Mais dans le cas (b), qui est générique, nous parlons plutôt de la fonction, de l'activité, et ici le masculin l'emporte même si, en fait, il y est question majoritairement, voire exclusivement de femmes.

Je termine cet exemple par une analyse d'un tout autre ordre et demande aux étudiants si l'ouvrier travaille est une bonne traduction des trois expressions en anglais. Ceci se fait par le biais de questions précises.

i) En anglais le phonème work est repris dans le sujet et dans le verbe. Pourrait-on dire le travailleur travaille en français? Avec le même effet? Que sait-on, dans ce cas, non pas de la personne qui travaille mais de la personne qui parle ? Ce qu'il faut remarquer, c'est que la connotation de worker est peut-être plus «neutre", moins marquée que le mot travailleur en français, où il existe une connotation (voir infra) syndicale et/ou communiste.

6 ii) Est-ce que le mot anglais et le mot français recouvrent les mêmes réalités ou phénomènes socio-économiques? Le mot anglais, ne serait-il pas plus général (voir shop worker, factory worker, office worker...) que le mot français qui, dans le langage courant, s'appliquerait plutôt aux travailleurs manuels, le mot employé étant plus usité dans les autres contextes?

\section{Exemple 3}

17 Telle langue oblige à donner certaines informations, telle autre permet de les retenir ou nécessite des explications supplémentaires. Comparons, de nouveau, l'anglais et le français, pour des termes courants qui précisent le sexe d'une personne mais qui contiennent ou taisent d'autres informations. Je me limite, ici, à la dénotation des termes et laisse volontairement de côté les questions de connotation (voir ci-après).

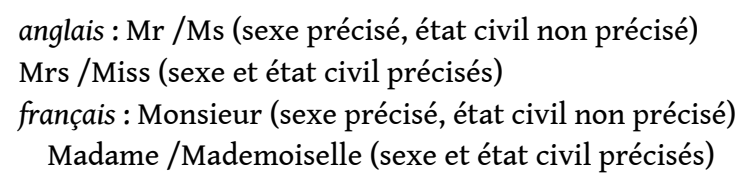

8 Comme nous le voyons, la langue anglaise permet une symétrie parfaite entre Mr et Ms ; que l'on soit homme ou femme on peut préciser son sexe tout en taisant son état civil. Le français de France ne le permet pas.

Comparons maintenant des termes qui précisent le sexe d'un individu et qui donnent, ou taisent, d'autres informations sur les liens de parenté et l'âge relatif.

anglais : woman/man; wife/husband; girl/boy ; daughter/son français : femme/homme; femme/mari ; fille/garçon ; fille/fils

Ici, de nouveau, la langue anglaise permet une symétrie parfaite. Il existe huit termes qui donnent tous une indication sur le sexe de la personne. En outre, quatre d'entre eux ( woman, man, girl, boy) indiquent une tranche d'âge relative, les quatre autres (wife, husband, daughter, son) donnent une indication sur une relation avec autrui (son époux/ épouse, ses parents). En français, il n'existe que six termes. Si le sexe est clairement indiqué et si, pour la gent masculine, les distinctions concernant l'âge et la relation avec autrui sont pareilles qu'en anglais, il n'en va pas de même pour la gent féminine. L'étudiant non averti, qui chercherait à traduire les mots femme ou fille en anglais hors 
contexte et/ou à l'aide d'un dictionnaire, risque de se tromper s'il ne remarque pas cette asymétrie et si le dictionnaire n'explique pas clairement que femme dans le sens d'épouse se traduit par wife, woman étant la traduction de femme par opposition à fille. Ici encore, je me limite à la dénotation des termes.

\subsection{Qu'est-ce qu'un dictionnaire?}

21 Après avoir passé les exemples ci-dessus en revue, je pose une deuxième grande question, à savoir qu'est-ce qu'un dictionnaire ? Là aussi, les réponses fusent, à commencer par un livre qui contient des mots, classés par ordre alphabétique. Lorsque je demande des précisions, ils me répondent qu'on peut également trouver des informations sur l'orthographe ; l'origine des mots (étymologie); des traductions (dictionnaires bilingues ou multilingues); la grammaire (parties du discours, catégories grammaticales) ; la phonétique (prononciation); des synonymes et des antonymes; des expressions et citations pertinentes ; des indications sur le bon usage des mots (formel, vulgaire...).

C'est à partir de ces différents éléments, fournis par les étudiants, que j'aborde d'autres aspects et que j'invite à une réflexion. Tout d'abord j'introduis, de manière très sommaire, les notions de dénotation (le sens objectif d'un mot), de connotation (jugement de valeur, image subjective d'un mot) et d'association (contexte, usage, citations). Pour essayer d'être le plus claire possible je donne quelques exemples, que je commente en faisant remarquer que les mots n'évoluent pas toujours de façon linéaire ou symétrique ; l'exemple (fig. 1) illustre bien la différence entre la dénotation et la connotation de couples de mots.

Figure 1. Exemple

\begin{tabular}{|l|l|l|}
\hline & dénotation & connotation \\
\hline \hline bachelor & célibataire de sexe masculin & célibataire de sexe masculin \\
\hline spinster & célibataire de sexe féminin & vieille fille \\
\hline
\end{tabular}

Une fois cette distinction entre dénotation et connotation comprise, je quitte, provisoirement, les mots pour revenir aux dictionnaires proprement dit. Pour ce faire je demande tout d'abord ce que signifient les deux termes « descriptif » et «normatif » et cherche à éliciter des définitions du genre: descriptif dit ce qui est (says what is) et normatif dit ce qui devrait être (says what should be). Une fois ces définitions bien comprises je demande "Un dictionnaire est-il descriptif ou normatif ?», en faisant très attention à la formulation, pour des raisons qui deviendront claires par la suite.

Pour faciliter la discussion, je donne souvent cet exemple : si, dans un dictionnaire, on dit que le mot shit est vulgaire, est-ce un commentaire descriptif ou normatif ? La discussion varie énormément d'un groupe à l'autre et je termine en faisant remarquer que la question elle-même est formulée de façon descriptive car j'aurais pu demander si un dictionnaire devait être descriptif ou normatif, c'est-à-dire poser la question de façon normative. Cependant, il n'y a aucune autre manière de poser la question et la structure même de la langue impose un choix. 
En conclusion, j'avance l'idée qu'un dictionnaire est à la fois un témoignage de la façon dont la langue est utilisée à un moment donné et un moyen de conserver et de prescrire la langue et les valeurs qu'elle exprime.

\subsection{Comment parler de choses nouvelles?}

Si le dictionnaire conserve et perpétue les valeurs d'une société, il ne peut que contenir des mots et des expressions existants. Pour décrire des phénomènes nouveaux, nommer des découvertes et des inventions, repenser des concepts, il faut créer, et ceci se fait en donnant de nouveaux sens aux mots existants, en inventant des néologismes et de nouvelles expressions, et en utilisant le langage existant dans de nouveaux contextes. Mais ce qu'il ne faut pas oublier, c'est qu'ils perpétuent également les valeurs de la culture dans laquelle ils sont véhiculés. Je prends un exemple dans Spender (1980:164-5), qui cite une expérience conduite par un psychologue, Witkin, et analyse les termes qu'il invente pour décrire les résultats.

Witkin cherche à vérifier si la perception qu'ont les hommes et les femmes du monde extérieur est la même ou différente. Il montre dans le même ordre à chaque personne (homme ou femme) participant à l'expérience une série d'images où l'on peut voir un stimulus contenu dans un cadre (lion dans une prairie, voiture sur une route vide, vase sur une table...) et l'invite à décrire ce qu'elle voit. Les images sont telles que l'on peut soit décrire le stimulus et le cadre comme formant un tout, soit distinguer le stimulus du cadre. Or, il se trouve que la majorité des femmes perçoit le stimulus et le cadre comme formant un tout, tandis que la majorité des hommes distingue le stimulus du cadre. Le problème se pose maintenant à Witkin de trouver des termes pour décrire ces résultats et c'est là que les valeurs dominantes se révèlent. Il choisit de nommer le comportement des hommes field independence, celui des femmes field dependence.

Je demande aux étudiants d'analyser ces deux expressions en essayant de faire abstraction de l'expérience de Witkin et j'essaie de leur montrer que si, sur le plan de la dénotation, les termes sont apparemment objectifs, en réalité ils sont très fortement connotés, l'image de l'indépendance renforçant les images positives de l'homme, celle de la dépendance renforçant les images négatives de la femme. Je termine cette partie en leur donnant une autre façon de nommer les comportements, celle proposée par Spender, et en leur demandant de l'analyser: les femmes font preuve de context awareness, les hommes de context blindness.

\section{Travail de préparation pour la séance suivante}

29 Les étudiants doivent chercher quatre groupes de mots en anglais et en français dans les dictionnaires suivants :

- dans le même dictionnaire français mais à des époques différentes

- dans le même dictionnaire anglais mais à des époques différentes

- dans différents dictionnaires français mais à la même époque

- dans différents dictionnaires anglais mais à la même époque Groupe I : erotic, pornographic, obscene/érotique, pornographique, obscène Groupe II : god, hell, phlogiston, purgatory/dieu, enfer, phlogistique, purgatoire Groupe III : black, white/noir, blanc 
Groupe IV : woman, man/femme, homme juristes, hommes politiques, experts en tout genre, qui étaient ou sont souvent pressés, qui n'étaient ou ne sont pas toujours sensibles aux ambiguïtés de la langue, qui vivent dans une société qui évolue et dont la langue n'est qu'un reflet imparfait des valeurs de l'époque. S'il est difficile pour un juriste anglais de s'y retrouver, à plus forte raison l'étudiant en droit non anglophone, qui doit être sensibilisé à ces questions, que ce soit celle de la dénotation ou de la connotation d'une expression, du registre ou du niveau de langue, du mot juste et de l'impact du choix lexical sur les intérêts du client.

Tout au long de l'année, que nous étudiions un point théorique, un arrêt, une question constitutionnelle ou institutionnelle, un problème dit de société sous un angle juridique, 
j'insiste sur ces aspects linguistiques - il s'agit, après tout, d'un séminaire de langue afin d'aider les étudiants à prendre conscience de ce qu'ils sont en train de dire, ou de lire. J'essaie de leur montrer que, dans leur futur métier, les approximations linguistiques ne suffisent pas, que ce soit dans une langue étrangère ou dans la leur propre. J'essaie également de leur montrer qu'une bonne maîtrise de la langue, quelle qu'elle soit, passe par cette sensibilisation, cette conscience de la relation étroite entre les mots qu'ils utilisent et les valeurs qu'ils véhiculent. Pour un linguiste, c'est une évidence. Pour un étudiant en droit peut-être moins.

\section{BIBLIOGRAPHIE}

Adams, Carol \& RAE Laurikietis. 1976. Messages and Images. Londres : Virago.

Bijeljac, Ranka \& Roland Breton. 1997. Du langage aux langues. Paris : Découvertes Gallimard. Bouscaren, Christian et Rosalind Greenstein. 1993. Les bases du droit anglais. Paris : Ophrys.

Cascino, Stéphanie. 1998. « Des mots pour cacher des maux ». Le Nouvel Observateur 1764, 27 août-2 septembre 1998, 22-24.

Fraser, Bill. 1997. «The new rhetoric. How discourse analysis can help translators ». Terminologie et Traduction 3, Commission européenne, Bruxelles, 149-179.

Garcia, Daniel. 1998. « Faute, vous avez dit faute, quelle faute ?». Le Nouvel Observateur 1763, 20-26 août 1998, 18-20.

Lakoff, Robin. 1975. Language and Woman's Place. New York \& Londres : Harper Colophon Books.

Loubier, Christiane. 1994. «L'implantation du français : du microcosme au macrocosme ». Terminologies nouvelles 12, 20-37.

Miller, Casey \& Kate Swift. 1979. Words and Women. Londres : Pelican Books.

Miller, Casey \& Kate Swift. 1981. The Handbook of Nonsexist Writing. New York : Barnes \& Noble Books.

Mounin, Georges. 1963. Les problèmes théoriques de la traduction. Paris : Gallimard.

Spender, Dale. 1980. Man Made Language. Londres \& Boston : Routledge and Kegan Paul.

Walter, Henriette. 1997. L'aventure des mots français venus d'ailleurs. Paris : Robert Laffont.

Yaguello, Marina. 1978. Les mots et les femmes. Paris : Payot.

Zander, Michael. 1994. The Law-Making Process. Londres : Butterworths.

Arrêts

DPP v Bull [1994] 4 All ER 411 (QBD)

Pepper v Hart [1983] 1 All ER 42 (HL)

Re Rowland [1963] 1 Ch. 1 (CA)

Dictionnaires 
Chambers's Twentieth Century Dictionary 1959, 1993

Collin's Cobuild English Language Dictionary 1987, 1995

Concise Oxford Dictionary 1911, 1929, 1934, 1951, 1964, 1976, 1982, 1990, 1995

Longman Dictionary of Contemporary English 1978, 1987, 1995

Longman Dictionary of the English Language 1984, 1991

Webster's New World Dictionary of the American Language 1980

Abrégé Littré 1990

Lexis (dictionnaire de la langue française, Larousse) 1975

Nouveau Petit Larousse Illustré 1952

Petit Larousse en couleurs 1972

Petit Larousse illustré 1915, 1997

Petit Robert 19911993

\section{NOTES}

1. cf. Zander (1994) notamment le chapitre $3: 105-189$, pour une discussion très intéressante sur l'interprétation de textes juridiques.

\section{RÉSUMÉS}

Ma pratique de traductrice ainsi que mon expérience de l'enseignement de l'anglais juridique me conduisent depuis plusieurs années à réfléchir à la fois sur les dictionnaires et sur les besoins linguistiques de mes étudiants, dans la perspective de leur future carrière. Le présent article présente l'introduction à mon enseignement et une partie de mes recherches.

My experience both as a professional translator and as a teacher of legal English has informed my research into dictionaries and made me think differently about the language needs of my students in the light of their future careers. This paper presents my introductory course and part of my research.

\section{INDEX}

Mots-clés : anglais juridique, connotation, dénotation, dictionnaire, interprétation, langage, langue, registre

Keywords : construction, dictionary, language, legal English, register 


\section{AUTEUR}

\section{ROSALIND GREENSTEIN}

Rosalind Greenstein est traductrice professionnelle et maître de conférences à l'université Paris 1 (Panthéon-Sorbonne), où elle enseigne l'anglais juridique en deuxième et troisième cycles de droit communautaire et droit des activités économiques. Elle est responsable du Centre de recherche en langues de spécialité et cultures (CERLAC). Elle est co-auteur de Les bases du droit anglais (Ophrys), co-traductrice de La richesse des nations d'Adam Smith (PUF) et de La trilogie d'Arnold Wesker (Gallimard). Rosalind.Greenstein@univ-paris1.fr 